Pacific Journal of Mathematic 


\title{
CHARACTERIZATION OF THE STEINER POINT
}

\author{
WALTER J. MEYeR
}

\begin{abstract}
Let $f$ be a mapping which associates with each compact convex set in $E^{n}$ a point of $E^{n}$. If $f$ is linear (in terms of the vector addition of convex sets), uniformly continuous and commutes with a substantial enough set of congruences of $E^{n}$, then $f(K)$ is the Steiner point of $K$ for all compact convex sets $K$.
\end{abstract}

Let $\mathscr{K}^{n}$ denote the collection of compact convex sets in $E^{n}$, endowed with the algebraic structure of vector addition [1, p. 29] and the topology of the Hausdorff metric [1, p. 34]. For each $A \in \mathscr{K}^{n}$ we define its Steiner point, denoted $s(A)$, by

$$
s(A)=\frac{1}{\sigma_{n}} \int_{S^{n-1}} h(A, u) u d m
$$

where $m$ is Lebesgue measure on $S^{n-1}$, the unit $n-1$ sphere centered at $\bar{O}$, the origin; $\sigma_{n}$ is the volume of the unit $n$-ball; $u$ is a variable vector ranging over $S^{n-1}$; and $h(A, u)$ is the support function of $A$, defined as $[1$, p. 23]:

$$
h(A, u)=\sup _{x \in A} x \cdot u .
$$

It is apparent that the mapping from $\mathscr{K}^{n}$ to $E^{n}$ which associates with each set $A$ the point $\mathrm{s}(A)$ is linear, continuous, and commutes with congruence transformations of $E^{n}$. In 1963 Grunbaum [3, p. 239] asked whether these properties characterize the Steiner point mapping. Shephard answered this affirmatively for the case $n=2$ [7]. K. A. Schmitt made an attempt [6] at the general problem but his paper contains an error (in proving the continuity of a certain function-see p. 390) which is apparently serious. Our contribution is the following.

THeorem. Let $T$ denote a set of orthogonal transformations of $E^{n}$ onto itself such that:

(a) $T$ is transitive on points of $S^{n-1}$, that is, if $u_{1}, u_{2} \in S^{n-1}$ then there exists $t$ in $T$ such that $t\left(u_{1}\right)=u_{2_{2}}$.

(b) For each $u_{0}$ in $S^{n-1}$ there exists a nonempty set

$$
T\left(u_{0}\right), T\left(u_{0}\right) \subset T,
$$

such that each $t$ in $T\left(u_{0}\right)$ fixes $u_{0}$ and, on the other hand, if $u$ is fixed by each $t$ in $T\left(u_{0}\right)$ then $u$ must be $\lambda u_{0}$ for some scalar $\lambda$. 
(1) If $f: \mathscr{K}^{n} \rightarrow E^{n}$ is linear, uniformly continuous and satisfies

$$
f(t(K))=t f(K)
$$

for each $t$ in $T$ and each $K$ in $\mathscr{K}^{n}$ then

$$
f(K)=\lambda s(K)
$$

for some fixed $\lambda$.

(2) If (*) also holds for each $K \in \mathscr{K}^{n}$ and $t$ a reflection in some point $b \neq \overline{0}$, then $\lambda=1$.

2. In outline the proof goes as follows. Instead of dealing with the space $\mathscr{K}^{n}$, we imbed $\mathscr{K}^{n}$ into a normed vector space $\mathscr{H}^{n}$. We extend $f$ to $f^{*}$ a linear continuous mapping of $\mathscr{H}^{n}$ into $E^{n}$ and then follow this by projection mappings to get $f_{i}^{*}$ a set of $n$ continuous linear functionals. We represent each of these as integrals with respect to measures $\mu_{i}$ and then show that the vector valued measure $\mu=\left(\mu_{1}, \cdots, \mu_{n}\right)$ has commutativity properties analogous to those assumed for $f$. To apply the commutativity properties we need to consider a derivative, $D \mu$, of $\mu$ with respect to Lebesgue measure $m$ and show it has certain commutativity properties. This derivative is a point to point mapping of $S^{n-1}$ to $E^{n}$ and for this reason we can characterize it from its commutativity properties. As this last fact is the point of our method we do this first and then proceed in the order indicated by the outline.

(1) LEMma 1. Let $f: S^{n-1} \rightarrow E^{n}$ be such that $f(t u)=t f(u)$ for each $u$ in $S^{n-1}$ and each $t$ in $T$, where $T$ is as described in the theorem. Then there exists $\lambda$ so that $f(u)=\lambda u$ for all $u$ in $S^{n-1}$.

Proof. Suppose $f\left(u_{0}\right) \neq \overline{0}$ for some $u_{0}$ in $S^{n-1}$. For each $t \in T\left(u_{0}\right)$,

$$
t f\left(u_{0}\right)=f\left(t u_{0}\right)=f\left(u_{0}\right)
$$

whence $f\left(u_{0}\right)=\lambda\left(u_{0}\right) u_{0}$ where $\lambda\left(u_{0}\right)$ is some scalar possibly depending on $u_{0}$. Now let $u_{1}$ be any other member of $S^{n-1}$. Then there exists $t$ in $T$ so that $t u_{0}=u_{1}$. Then

$$
\begin{aligned}
\lambda\left(u_{1}\right) u_{1}=f\left(u_{1}\right) & =f\left(t u_{0}\right)=t f\left(u_{0}\right) \\
& =t\left[\lambda\left(u_{0}\right) u_{0}\right]=\lambda\left(u_{0}\right) t u_{0}=\lambda\left(u_{0}\right) u_{1}
\end{aligned}
$$

whence $\lambda\left(u_{1}\right)=\lambda\left(u_{0}\right)$.

(2) The mapping $A \rightarrow h(A, u)$ is an isomorphism of $\mathscr{K}^{n}$ onto 
the space of support functions of compact convex sets in $E^{n}$ [1, p. 26, 29.]. Because support functions are positively homogeneous we can consider instead their restrictions to $S^{n-1}$ and define an isomorphism of $\mathscr{K}^{n}$ onto this class in the obvious way. Let $\mathscr{H}^{n}$ denote the set of all differences of these restricted support functions. Because the support functions are closed under addition and scalar multiplication, $\mathscr{X}^{n}$ is a vector space in which $\mathscr{K}^{n}$ is isomorphically imbedded. If we provide $\mathscr{X}^{n}$ with the supremum norm, this isomorphism is an isometry [1, p. 35]. (A slightly different description of this can be found in [4].) Now we can extend $f$ to $f^{*}$, a linear function on $\mathscr{C}^{n}$ by defining

$$
f^{*}(g)=f\left(h_{1}\right)-f\left(h_{2}\right)
$$

whenever $g=h_{1}-h_{2}$ where $h_{1}, h_{2}$ are restricted support functions. The uniform continuity of $f$ yields continuity for $f^{*}$. Composing this with the projection mapping onto the $i^{\text {th }}$ coordinate of $E^{n}$ we have $f_{i}^{*}$, a real continuous liner functional on the space $\mathscr{C}^{n}$. Now $\mathscr{C}^{n}$ is dense in the space of real continuous functions on $S^{n-1}$ $\left[2\right.$, p. 10]. Let $f_{i}^{*}$ be extended to be defined on this larger space so that we may apply the Riesz Representation Theorem [5, p. 131] to get, for $i=1,2, \cdots, n$, real regular Borel measures $\mu_{i}$ on $S^{n-1}$ with the property that:

$$
f_{i}^{*}(g)=\int_{S^{n-1}} g(u) d \mu_{i}(u) .
$$

To compactify our notation we can write:

$$
f^{*}(g)=\int_{S^{n-1}} g(u) d \mu(u)
$$

where $\mu$ denotes the vector valued measure defined by

$$
\mu(E)=\left(\mu_{1}(E), \cdots, \mu_{n}(E)\right) .
$$

(3) To investigate the effect of the hypothesis requiring commutativity with certain orthogonal transformations, note that if $t$ is orthogonal,

$$
\begin{aligned}
h(t A, u) & =h\left(t A, t\left(t^{-1} u\right)\right) \\
& =\sup _{x \in t A}\left[x \cdot t\left(t^{-1} u\right)\right] \\
& =\sup _{y \in A}\left[t y \cdot t\left(t^{-1} u\right)\right] \\
& =\sup _{y \in A}\left[y \cdot t^{-1} u\right] \\
& =h\left(A, t^{-1} u\right) .
\end{aligned}
$$


Consequently for each $g \in \mathscr{H}^{n}$

$$
f^{*}\left[g_{t^{\prime}}\right]=t f^{*}[g]
$$

where $g_{t}(u)=g\left(t^{-1} u\right)$. In terms of our integral representations,

$$
\begin{aligned}
t \int_{S^{n-1}} g(u) d \mu(u) & =\int_{S^{n-1}} g\left(t^{-1} u\right) d \mu(u) \\
& =\int_{S^{n-1}} g(u) d \mu(t u) .
\end{aligned}
$$

But since $t$ is linear and continuous, for any function $g$,

$$
t \int_{S^{n-1}} g(u) d \mu(u)=\int_{S^{n-1}} g(u) d(t \mu)(u) .
$$

Therefore, for each $g \in \mathscr{H}^{n}$ :

$$
\int_{S^{n-1}} g(u) d(t \mu)(u)=\int_{S^{n-1}} g(u) d \mu(t u) .
$$

Since $\mathscr{H}^{n}$ is dense in the space of continuous functions on $S^{n-1}$, the last equation shows that for each Borel set $E$,

$$
t \mu(E)=\mu(t E) \text {. }
$$

(4) The next step is to consider a Lebesgue decomposition of $\mu$ with respect to $m$, and then to show that the derivative of the components of $\mu$ with respect to $m$ and a suitable Vitali covering have the appropriate commutativity property. For each of $\mu_{1}$, $\mu_{2}, \cdots, \mu_{n}$ write its Lebesgue decomposition:

$$
\mu_{i}(E)=u_{i}^{*}(E)+\mu_{i}^{* *}(E)
$$

[8, p. 187] where $\mu_{i}^{*}$ is absolutely continuous with respect to $m$ and $\mu_{i}^{* *}$ is singular with respect to $m$. We can then write

$$
\mu(E)=\mu^{*}(E)+\mu^{* *}(E)
$$

where $\mu^{*}$ and $\mu^{* *}$ are vector measures whose respective components are the $\mu_{i}^{*}$ and $\mu_{i}^{* *}$.

We will now consider derivatives of $\mu^{*}$ and $\mu^{* *}$ with respect to Lebesgue measure $m$ and the Vitali covering of $S^{n-1}$ consisting of spherical caps of the form $\left\{x \in S^{n-1} \mid x \cdot a \geqq r\right\}, o<r<1, a \in S^{n-1}$ using the derivation process described below [8, p. 221].

Definition. (1) If $E_{1}, E_{2}, \ldots$ is a sequence of Borel sets, they are said to converge regularly to $x$ provided there exists a sequence of Vitali sets $A_{1}, A_{2}, \ldots$ such that: $x \in A_{i}$ and $E_{i} \subset A_{i}$ for each $i$, 
$m\left(A_{i}\right) \rightarrow 0$, and there exists a fixed constant $c>0$ such that $m\left(E_{i}\right) \geqq c m\left(A_{i}\right)$.

(2) Let $\nu$ be any countably additive set function (real or vector valued). Then

$$
D \nu(x)=\lim _{i \rightarrow \infty} \frac{\nu\left(E_{i}\right)}{m\left(E_{i}\right)}
$$

where $E_{i}$ is any sequence reqularly converging to $x$, provided the limit exists and is independent of the choice of the $E_{i}$.

LEMMA 2. Let $\nu$ be any vector valued measure on $S^{n-1}$ where $\nu(t E)=t \nu(E)$ holds for a set of orthogonal transformations which is transitive on $S^{n-1}$. Then $D \nu(x)$ exists for all $x$ and $D \nu(t x)=t D \nu(x)$.

Proof. First observe that our Vitali system is closed under orthogonal transformations. Moreover, so is the family $\mathscr{B}$ of Borel sets. For this reason, and because $m(t E)=m(E)$ for any orthogonal $t$, if $E_{i}$ converge regularly to $x, t E_{i}$ converge regularly to $t x$. Furthermore, any sequence converging regularly to $t x$ arises this way.

Now suppose $D \nu(x)$ exists and $y$ is any other point of $S^{n-1}$. For some $t$ we have $t x=y$ and:

$$
\begin{aligned}
t D \nu(x) & =t \lim _{i \rightarrow \infty} \frac{\nu\left(E_{i}\right)}{m\left(E_{i}\right)} \\
& =\lim _{i \rightarrow \infty} \frac{t \nu\left(E_{i}\right)}{m\left(E_{i}\right)} \\
& =\lim _{i \rightarrow \infty} \frac{\nu\left(t E_{i}\right)}{m\left(t E_{i}\right)} \\
& =D \nu(t x) .
\end{aligned}
$$

However $D \nu(x)$ exists except for a set of Lebesgue measure $0[8$, p.222]. This proves the lemma.

Now, to make the connection between this derivative and (**), we have $[8, p .222]$ :

$$
\mu(E)=\mu^{* *}(E)+\int_{E} D \mu(u) d m .
$$

$D \mu(u)$ satisfies the hypotheses of Lemma 1 so this becomes:

$$
\mu(E)=\mu^{* *}(E)+\int_{E} \lambda u d m .
$$

Comparing this with $\left({ }^{*}\right)$ we see that the first assertion of the Theorem will follow if $u^{* *}$ is identically $\overline{0}$. 
The measure $\int_{E} \lambda u d m(u)$ is regular since $m$ is regular, and also inherits the commutativity properties:

$$
\begin{aligned}
\int_{t E} \lambda u d m(u) & =\int_{E} \lambda(t u) d m(t u) \\
& =\int_{E} t(\lambda u) d m(u) \\
& =t \int_{E} \lambda u d m(u) .
\end{aligned}
$$

Consequently $\mu^{* *}$ is regular and commutes with all $t$ in $T$. Being a singular measure, $D \mu^{* *}(u)=\overline{0}$ except for a set of Lebesgue measure 0 . But by Lemma 2, this means that $D \mu^{* *}(u)=\overline{0}$ everywhere. Now the following lemma completes the proof of the first assertion of the theorem.

Lemma 3. Let $\mu$ be vector valued measure on $S^{n-1}$ such that:

(1) $D \mu(x)=\overline{0}$ for all $x$ in $S^{n-1}$.

(2) $\mu$ is regular.

Then $\mu$ is identically $\overline{0}$.

Proof. The idea of this long-winded proof is rather simple. Because of the regularity, it is enough to consider sets of positive Lebesgue measure. If $E$ is a set of positive Lebesgue measure for which $|\mu(E)|=\alpha m(E)>0$ then we use a Heine-Borel type argument (partitioning sets into subsets of equal Lebesgue measure) to find a nested sequence of subsets $E \supset E_{1} \supset E_{2} \cdots$ where

$$
\left|\mu\left(E_{i}\right)\right|>\alpha m\left(E_{i}\right)>0,
$$

and where the $E_{i}$ converge regularly to a point $a$. Then

$$
\left|D \mu^{\prime}(a)\right| \geqq \alpha .
$$

Before proceeding we need some preliminaries. Let $E^{n-1}$ denote a subspace of $E^{n}$. If $x \in S^{n-1}$ we give it a coordinate representation $x=\left(x_{1}, \cdots, x_{n}\right)$ where $x_{1}, \cdots, x_{n-1}$ are coordinates with respect to a fixed orthogonal basis of $E^{n-1}$ and $x_{n}$ is measured along the orthogonal complement of $E^{n-1}$. Denote by $N_{\varepsilon}$ the set $\left\{x \in S^{n-1} \mid x_{n} \geqq \varepsilon\right\}$. Henceforth let $\varepsilon$ be fixed and suppose $0<\varepsilon<1$. Let $p$ denote the projection map onto $E^{n-1}$ in the direction of the axis of the $n^{t h}$ coordinate. We will show that subsets of $N_{\varepsilon}$ have $\mu$ measure $\overline{0}$. The result then follows by covering $S^{n-1}$ by finitely many caps of this type. 
We now define a net on $N_{\varepsilon}$, that is, a countable collection of partitions, $\Omega_{1}, \Omega_{2}, \ldots$, of $N_{\varepsilon}$ into countably many Borel sets such that: $\Omega_{i+1}$ is a refinement of $\Omega_{i}$ and where, if $B_{i} \in \Omega_{i}$ and $B_{1} \supset B_{2} \supset \ldots$ we have $\bigcap_{1}^{\infty} \bar{B}_{i}$ is a single point. Rudin [5, p. 49,50] describes a net $\left\{\Omega_{i}^{\prime}\right\}$ for $E^{n-1}$ which has the further property that each set of $\Omega_{i}^{\prime}$ is an $n-1$ cube of side $2^{-i}$ and $n-1$ dimensional Lebesgue measure $2^{-i(n-1)}$ and is composed of $2^{n-1}$ sets from $\Omega_{i+1}^{\prime}$. By intersecting all these cubes with $p\left(N_{\varepsilon}\right)$ we get a net for $p\left(N_{\varepsilon}\right)$. Those cubes contained entirely in $p\left(N_{\varepsilon}\right)$ (and are not truncated by intersection with $p\left(N_{\varepsilon}\right)$ ) are called proper. We can "lift" this net to a net for $N_{\varepsilon}$ by the inverse mapping $p^{-1}$. Call these partitions $\Omega_{1}, \Omega_{2}, \cdots$, and distinguish by the term proper those cubes which arise from proper cubes in $p\left(N_{\varepsilon}\right)$.

Now define a new measure $\bar{m}$ on $S^{n-1}$ by

$$
\bar{m}(E)=m^{\prime}(p(E))
$$

where $m^{\prime}$ is Lebesgue measure in $E^{n-1}$. Since $m(E)=\int_{p(E)} \frac{1}{x_{n}} d m^{\prime}$ and $1 \leqq \frac{1}{x_{n}} \leqq \frac{1}{\varepsilon}$ we have :

$$
\bar{m}(E)=m^{\prime}(p(E)) \leqq m(E) \leqq \frac{1}{\varepsilon} m^{\prime}(p(E))=\frac{1}{\varepsilon} \bar{m}(E) .
$$

Consequently $m$ and $\bar{m}$ are absolutely continuous with respect to one another and our earlier defined Vitali system for $m$ is also one for $\bar{m}$.

Now if we calculate a derivative $\bar{D} \mu$ of $\mu$ with respect to $\bar{m}$ in the usual way, with respect to the same Vitali system, namely,

$$
\bar{D} \mu(x)=\lim _{i \rightarrow \infty} \frac{\mu\left(E_{i}\right)}{\bar{m}\left(E_{i}\right)}
$$

we have:

$$
|\bar{D} \mu(x)| \leqq \frac{1}{\varepsilon}|D \mu(x)|
$$

Hypothesis 1 then gives $\bar{D} \mu(x)=\overline{0}$.

The machinery for the proof has now been set up. By regularity, it is enough to show $\mu$ is $\overline{0}$ on open sets. But open sets are disjoint unions of countably many proper cubes $B_{i}$ from $\Omega_{1} \cup \Omega_{2} \cup \cdots$ where $\bar{m}\left(B_{i}\right)=2^{-j(n-1)}$ whenever $B_{i} \in \Omega_{j}$ (this follows easily from the corresponding result for the net $\left\{\Omega_{i}^{\prime}\right\}$ for $\left.E^{n-1}[5, \mathrm{p} .49,50]\right)$. Therefore we need only show that $\mu$ is $\overline{0}$ on proper cubes. Suppose the contrary, that is, there exists a proper cube $B_{0} \in \Omega_{k}$ with $\left|\mu\left(B_{0}\right)\right|>\alpha \bar{m}\left(B_{0}\right)$ 
for some positive $\alpha$. Since $B_{0}$ is the union of $2^{n-1}$ proper cubes from $\Omega_{k+1}$, each with the same $\bar{m}$ measure, we can find $B_{1} \in \Omega_{k+1}$ where

$$
\left|\mu\left(B_{1}\right)\right| \geqq \frac{1}{2^{n-1}}\left|\mu\left(B_{0}\right)\right|>\alpha \frac{\bar{m}\left(B_{0}\right)}{2^{n-1}}=\alpha \bar{m}\left(B_{1}\right) .
$$

Proceeding in this way we find $B_{0} \supset B_{1} \supset B_{2} \cdots$ with $\frac{\left|\mu\left(B_{i}\right)\right|}{\bar{m}\left(B_{i}\right)} \geqq \alpha>0$. Now $\bigcap_{1}^{\infty} \bar{B}_{i}$ is a single point, say $a$. If we can show that the $B_{i}$ converge regularly to $a$, then $|\bar{D} \mu(a)| \geqq \alpha>0$, a contradiction.

To establish the regular convergence, we examine the numbers $f(i)=\bar{m}\left(\bar{S}\left(a, r_{i}\right)\right) / \bar{m}\left(B_{i}\right)$ where $\bar{S}\left(a, r_{i}\right)$ is the smallest closed cap centered at a containing $B_{i}$. It is sufficient to show that $\limsup _{i \rightarrow \infty} f(i)<\infty$. The proof of this, although straight-forward, is tedious and we omit the details. This concludes the proof of Lemma 3 and the first part of the Theorem.

Concerning the second assertion of the theorem, suppose $t$ is a reflection through the point $b$ where $b \neq \overline{0}$ and suppose that $f$ commutes also with $t$. By part $1, f$ is of the form $\lambda s$ so $\lambda s(t b)=t[\lambda s(b)]$. But $t$ has $b$ as its one and only fixed point so this gives $\lambda s(b)=$ $t[\lambda s(b)]$ whence $\lambda s(b)=b$. But $s(b)=b \neq 0$ so $\lambda=1$.

REMARKs. For $n \leqq 2$ the set $T$ of the theorem must include indirect (orientation reversing) congruences if it is to satisfy the hypotheses on $T$. For $n=1$ the only orthogonal transformation is reflection in $\overline{0}$. If we drop the commutativity with this reflection and assume instead commutativity with all translations we do not get the result of the theorem. The mapping $[a, b] \rightarrow b$ is a counterexample. For $n=2$, although our method of proof does not yield the representation $f(K)=\lambda s(K)$ if we only require commutativity with rotations, we have been able to deduce the same representation by connecting the problem with the uniqueness of Haar measures on $S^{1}$.

Concerning the continuity requirements, Shephard [7] has shown that continuity (as opposed to uniform continuity) suffices when $n=2$. If $n=1$ even continuity is unnecessary. For we can represent a closed interval as a pair of real variables representing the endpoints and then $f$ becomes a linear function of these variables and we can write $f(x, y)=\alpha x+\beta y$. We can easily show $\alpha=\beta$ by using commutativity with reflections, and this gives the result. 


\section{REFERENCES}

1. T. Bonnesen and W. Fenchel, Theorie der Konvexen Körper, Chelsea Publishing Company, New York, 1948.

2. W. Fenchel and B. Jessen, Mengen funktionen und konvexe Korper, Det. Kgl. Danske Videnskab, Selskab, Math. -fys. Medd. 16 (1938), 3.

3. B. Grünbaum, Measures of symmetry for convex sets, in Convexity, Proceedings of Symposia in Pure Mathematics, Vol. vii, Amer. Math. Soc., Providence, 1963.

4. H. Radstrom, An embedding theorem for spaces of convex sets, Proc. Amer. Math. Soc. (1952) 165-169.

5. W. Rudin, Real and Complex Analysis, McGraw-Hill, 1966.

6. K. A. Schmitt, Kennzeichnung des Steinerpunktes Konvexer Körper, Math. Zeit. 105 (1968) 387-392.

7. G. C. Shephard, A uniqueness theorem for the Steiner point of a convex region, J. London Math. Soc. 43 (1968), 439-444.

8. G. E. Shilov and B. L. Gurevich, Integral, Measure and Derivative: A Unified Approach, trans. by R. A. Silverman, Prentice-Hall, Inc., 1966.

Received October 20, 1969. This work forms part of the author's Ph. D. dissertation at the University of Wisconsin, submitted in August 1969.

\section{ADELPHI UNIVERSITY}

GARDEN City, NeW YoRK 



\section{PACIFIC JOURNAL OF MATHEMATICS}

\section{EDITORS}

\author{
H. SAMELSON \\ Stanford University \\ Stanford, California 94305 \\ Richard Pierce \\ University of Washington \\ Seattle, Washington 98105
}

\author{
J. DugundJI \\ Department of Mathematics \\ University of Southern California \\ Los Angeles, California 90007 \\ RICHARD ARENS \\ University of California \\ Los Angeles, California 9.0024
}

\section{ASSOCIATE EDITORS}

\begin{tabular}{|c|c|}
\hline E. F. BECKENBACH & K. YoshidA \\
\hline \multicolumn{2}{|c|}{ SUPPORTING INSTITUTIONS } \\
\hline UNIVERSITY OF BRITISH COLUMBIA & STANFORD UNIVERSITY \\
\hline CALIFORNIA INSTITUTE OF TECHNOLOGY & UNIVERSITY OF TOKYO \\
\hline UNIVERSITY OF CALIFORNIA & UNIVERSITY OF UTAH \\
\hline MONTANA STATE UNIVERSITY & WASHINGTON STATE UNIVERSITY \\
\hline UNIVERSITY OF NEVADA & UNIVERSITY OF WASHINGTON \\
\hline NEW MEXICO STATE UNIVERSITY & * \\
\hline OREGON STATE UNIVERSITY & AMERICAN MATHEMATICAL SOCIETY \\
\hline UNIVERSITY OF OREGON & CHEVRON RESEARCH CORPORATION \\
\hline OSAKA UNIVERSITY & TRW SYSTEMS \\
\hline UNIVERSITY OF SOUTHERN CALIFORNIA & NAVAL WEAPONS CENTER \\
\hline
\end{tabular}

The Supporting Institutions listed above contribute to the cost of publication of this Journal, but they are not owners or publishers and have no responsibility for its content or policies.

Mathematical papers intended for publication in the Pacific Journal of Mathematics should be in typed form or offset-reproduced, (not dittoed), double spaced with large margins. Underline Greek letters in red, German in green, and script in blue. The first paragraph or two must be capable of being used separately as a synopsis of the entire paper. The editorial "we" must not be used in the synopsis, and items of the bibliography should not be cited there unless absolutely necessary, in which case they must be identified by author and Journal, rather than by item number. Manuscripts, in duplicate if possible, may be sent to any one of the four editors. Please classify according to the scheme of Math. Rev. Index to Vol. 39. All other communications to the editors should be addressed to the managing editor, Richard Arens, University of California, Los Angeles, California, 90024.

50 reprints are provided free for each article; additional copies may be obtained at cost in multiples of 50 .

The Pacific Journal of Mathematics is published monthly. Effective with Volume 16 the price per volume (3 numbers) is $\$ 8.00$; single issues, $\$ 3.00$. Special price for current issues to individual faculty members of supporting institutions and to individual members of the American Mathematical Society: $\$ 4.00$ per volume; single issues $\$ 1.50$. Back numbers are available.

Subscriptions, orders for back numbers, and changes of address should be sent to Pacific Journal of Mathematics, 103 Highland Boulevard, Berkeley, California, 94708.

PUBLISHED BY PACIFIC JOURNAL OF MATHEMATICS, A NON-PROFIT CORPORATION

Printed at Kokusai Bunken Insatsusha (Internatıonal Academic Printing Co., Ltd.), 7-17, Fujimi 2-chome, Chiyoda-ku, Tokyo, Japan. 


\section{Pacific Journal of Mathematics}

\section{Vol. 35, No. $3 \quad$ November, 1970}

John D. Arrison and Michael Rich, On nearly commutative degree one algebras . . . 533

Bruce Alan Barnes, Algebras with minimal left ideals which are Hilbert spaces . . . . 537

Robert F. Brown, An elementary proof of the uniqueness of the fixed point index . . . 549

Ronn L. Carpenter, Principal ideals in F-algebras .................... 559

Chen Chung Chang and Yiannis (John) Nicolas Moschovakis, The Suslin-Kleene

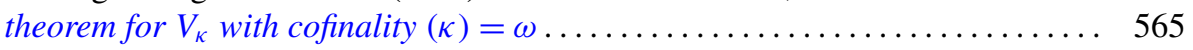

Theodore Seio Chihara, The derived set of the spectrum of a distribution

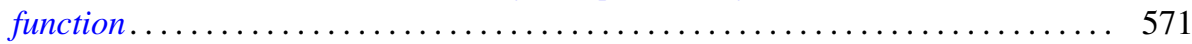

Tae Geun Cho, On the Choquet boundary for a nonclosed subspace of $C(S) \ldots \ldots \quad 575$

Richard Brian Darst, The Lebesgue decomposition, Radon-Nikodym derivative,

conditional expectation, and martingale convergence for lattices of sets .......

David E. Fields, Dimension theory in power series rings . . . . . . . . . . . .

Michael Lawrence Fredman, Congruence formulas obtained by counting

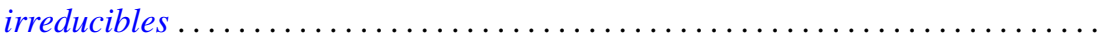

John Eric Gilbert, On the ideal structure of some algebras of analytic functions.....

G. Goss and Giovanni Viglino, Some topological properties weaker than

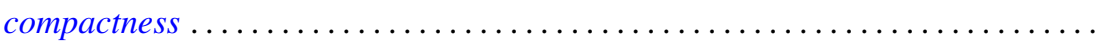

581

601

625

George Grätzer and J. Sichler, On the endomorphism semigroup (and category) of

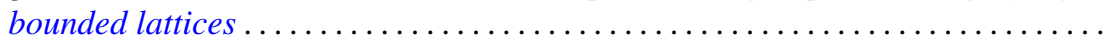

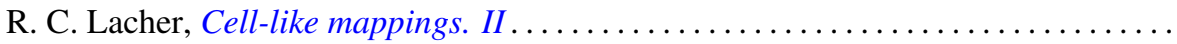

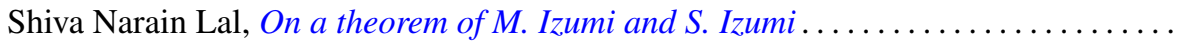

661

Howard Barrow Lambert, Differential mappings on a vector space ...............

Richard G. Levin and Takayuki Tamura, Notes on commutative power joined

semigroups.

Robert Edward Lewand and Kevin Mor McCrimmon, Macdonald's theorem for quadratic Jordan algebras.

J. A. Marti, On some types of completeness in topological vector spaces ....

Walter J. Meyer, Characterization of the Steiner point

717

Saad H. Mohamed, Rings whose homomorphic images are $q$-rings ...

727

Thomas V. O'Brien and William Lawrence Reddy, Each compact orientable surface

of positive genus admits an expansive homeomorphism ...

737

Robert James Plemmons and M. T. West, On the semigroup of binary relations...

743

Calvin R. Putnam, Unbounded inverses of hyponormal operator . .

755

William T. Reid, Some remarks on special disconjugacy criteria for differential

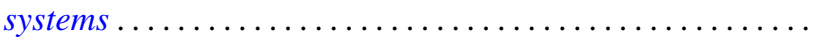

C. Ambrose Rogers, The convex generation of convex Borel sets in euclidean

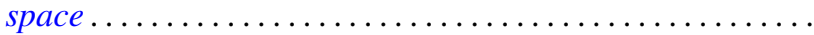

S. Saran, A general theorem for bilinear generating functions .

S. W. Smith, Cone relationships of biorthogonal systems ......

Wolmer Vasconcelos, On commutative endomorphism rings ....

795

Vernon Emil Zander, Products of finitely additive set functions from Orlicz

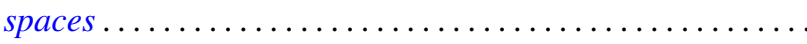

G. Sankaranarayanan and C. Suyambulingom, Correction to: "Some renewal

theorems concerning a sequence of correlated random variables" .

Joseph Zaks, Correction to: "Trivially extending decompositions of $E^{n}$ ”....... 805

Dong Hoon Lee, Correction to: "The adjoint group of Lie groups" ............ 805

James Edward Ward, Correction to: "Two-groups and Jordan algebras". 